\title{
OPEN Nerve growth factor and glutamate increase the density and expression of substance $P$-containing nerve fibers in healthy human masseter muscles
}

\author{
Abdelrahman M. Alhilou ${ }^{1,2 \bowtie}$, Akiko Shimada ${ }^{3}$, Camilla I. Svensson ${ }^{4}$, Peter Svensson ${ }^{5}$, \\ Malin Ernberg ${ }^{2}$, Brian E. Cairns ${ }^{6}$ \& Nikolaos Christidis ${ }^{2}$
}

Nocifensive behavior induced by injection of glutamate or nerve growth factor (NGF) into rats masseter muscle is mediated, in part, through the activation of peripheral NMDA receptors. However, information is lacking about the mechanism that contributes to pain and sensitization induced by these substances in humans. Immunohistochemical analysis of microbiopsies obtained from human masseter muscle was used to investigate if injection of glutamate into the NGF-sensitized masseter muscle alters the density or expression of the NMDA receptor subtype 2B (NR2B) or NGF by putative sensory afferent (that express SP) fibers. The relationship between expression and pain characteristics was also examined. NGF and glutamate administration increased the density and expression of NR2B and NGF by muscle putative sensory afferent fibers $(P<0.050)$. This increase in expression was greater in women than in men $(P<0.050)$. Expression of NR2B receptors by putative sensory afferent fibers was positively correlated with pain characteristics. Results suggest that increased expression of peripheral NMDA receptors partly contributes to the increased pain and sensitivity induced by intramuscular injection of NGF and glutamate in healthy humans; a model of my ofascial temporomandibular disorder (TMD) pain. Whether a similar increase in peripheral NMDA expression occurs in patients with painful TMDs warrants further investigation.

Masticatory muscle pain (myalgia/myofascial pain) is one of the most prevalent symptoms among temporomandibular disorders (TMD) ${ }^{1}$. TMD-myalgia is localized muscle pain that is exacerbated by jaw muscle function and palpation ${ }^{2}$. This type of chronic pain is more common in women than men $^{3,4}$. The pathophysiological mechanism involved in TMD-myalgia as well as the sex-related differences are not yet well understood. To investigate this aspect and to develop effective treatments for painful TMDs, standardized experimental pain models that mimic the clinical characteristics of this disorder are required ${ }^{5-7}$. For TMD-myalgia, several exogenous experimental pain models that use injections of different substances such as glutamate, serotonin, acidic saline, hypertonic saline and nerve growth factors (NGF) have been proposed ${ }^{8-14}$. Both glutamate and NGF have advantages over other pain models where experimental injection of these substances alone or in combination into healthy human masseter muscle produced similar pain characteristics involved in TMD myalgia ${ }^{7,15,16}$. However, the cellular and molecular mechanisms involved in these models are still not well addressed in the literature, especially for humans.

Substance P (SP) and NGF are important neurotransmitters/modulators that have been shown to mediate inflammatory hyperalgesia ${ }^{17,18}$. During inflammation NGF, by the help of cytokines, is thought to be released

\footnotetext{
${ }^{1}$ Department of Restorative Dentistry, College of Dentistry, Umm Al-Qura University, Makkah al Mukarramah, Saudi Arabia. ${ }^{2}$ Division of Oral Diagnostics and Rehabilitation, Department of Dental Medicine, Karolinska Institutet, and Scandinavian Center for Orofacial Neurosciences (SCON), Box 4064, 14104 Huddinge, Sweden. ${ }^{3}$ Department of Geriatric Dentistry, Osaka Dental University, Osaka, Japan. ${ }^{4}$ Department of Physiology and Pharmacology, Center for Molecular Medicine, Karolinska Institutet, Stockholm, Sweden. ${ }^{5}$ Department of Dentistry and Oral Health, Aarhus University, and Center for Orofacial Neurosciences (SCON), Aarhus, Denmark. ${ }^{6}$ Faculty of Pharmaceutical Sciences, University of British Columbia, Vancouver, Canada. ${ }^{\square}$ email: amhilou@uqu.edu.sa
} 
from different cells ${ }^{19-23}$. NGF binds to tyrosine kinase A (TrkA) receptors on nociceptive endings ${ }^{24}$, which in turn is retrogradely transported from the site of inflammation to the dorsal root ganglion leading to increased SP transcription $^{25}$. In rats, experimentally induced inflammation of the gastrocnemius and soleus muscle increased the density of nerve fibers (mainly perivascular fibers) expressing SP or NGF ${ }^{26}$. However, administration of NGF alone has been shown to induce sensory hypersensitivity without any inflammatory response ${ }^{27}$. Therefore, it is of interest to know if these neurotransmitters also are involved in experimentally induced masseter myalgia. Especially since the majority of TMD does not show signs of inflammatory changes ${ }^{28,29}$.

Myalgia can be initiated by noxious stimuli (mechanical or chemical) applied to the muscle, which in turn can activate specific receptors in the muscle nerve fibers as a consequence of the elevation of algogenic substances, such as glutamate ${ }^{30}$. In patients suffering from TMD-related myalgia, the glutamate level is elevated in the masseter muscle interstitial fluid as well as in the saliva and plasma ${ }^{31,32}$. In rats, injection of either NGF or glutamate induces muscle sensitization, in part, through the activation of peripheral N-methyl-D-aspartate (NMDA) receptors (glutamate receptors) ${ }^{8,11,12,33}$. While these findings in animals suggest an interaction between NGF and NMDA receptors, their interaction in human muscle is still unknown. Therefore, this study aimed to investigate the effect of NGF and glutamate injections on the density of nerve fibers in general and on the density of putative sensory afferent (that express SP) nerve fibers as well as their expression of NMDA-receptor subtype $2 \mathrm{~B}$ (NR2B) and NGF in human masseter muscle, to correlate expression with pain characteristics, and to determine any possible sex-related differences in these effects of combined injections.

\section{Methods}

Participants. Advertisements were posted on the Aarhus University campus (Denmark) and on an internet-page (https://aucobe.sona-systems.com/). In total, 15 healthy women and 15 age-matched healthy men (mean(SD) 24(4) years of age) were recruited. Screening for TMD was accomplished by using the diagnostic criteria for TMD (DC/TMD) ${ }^{2}$. Exclusion criteria were TMD related pain, facial pain, palpatory tenderness, neurological disorder, inflammatory diseases, fibromyalgia, whiplash-associated disorders, neuropathic disorders or pregnancy. Participants were informed not to use anti-inflammatory or analgesic medication for at least $24 \mathrm{~h}$ before the procedures and until the end of the experiment. All participants gave informed consent. The experiment followed the guidelines of the Helsinki declaration and was approved by the ethical committee in Aarhus, Demark (Midtjylland, approval No. 1-10-72-199-15).

Study design. The study comprised three sessions (day 0, day 3, and day 4). On day 0, $0.4 \mathrm{~mL}$ NGF (25 $\mu \mathrm{g} /$ $\mathrm{mL}$ sterile solution; Skanderborg Apotek, Aarhus, Denmark) was injected into the masseter muscle on the experimental side (left side). On day 3, glutamate (1 M, $0.2 \mathrm{~mL}$ sterile solution; Skanderborg Apotek, Aarhus, Denmark) was injected into the masseter on the same side. The solution was previously tested to have its effect on the nerve fibers through the activation of peripheral NMDA receptors and not due to its hypertonicity ${ }^{8}$. The technique followed for injections (glutamate or NGF) was standard and precise as previously described ${ }^{34}$. Aqueous solution was used as a solvent for both injections. Masseter microbiopsies were obtained both on day 0 from the control side and on day 4 from the experimental (injection) side as has been previously described in detail $^{35,36}$. In each session, pressure pain threshold (PPT), chewing-evoked pain and temporal summation pain were recorded from both sides before (e.g. baseline) and $5 \mathrm{~min}$ after the injections. Pain intensity at rest was recorded directly after injections on day 0 and 3 (Fig. 1a). The results from these examinations as well as DC/ TMD results have been presented elsewhere ${ }^{15}$.

Immunohistochemistry and image analysis. Biopsy samples were fixed over-night at $4{ }^{\circ} \mathrm{C}$, with $4 \%$ paraformaldehyde. Prior freezing $\left(-80^{\circ} \mathrm{C}\right)$, samples were rinsed with phosphate-buffered saline (PBS) and dehydrated first with a $20 \%$ and then with a $40 \%$ sucrose solution. Sliced sections were treated with $10 \%$ normal donkey serum in PBS for $1 \mathrm{~h}$, and then incubated for $24 \mathrm{~h}$ with primary antibodies against PGP 9.5 (1:250, anti-human mouse monoclonal, ABCAM Inc, Cambridge, England, ab72911), NR2B (1:200, anti-human rabbit polyclonal, ABCAM Inc, Cambridge, England, ab65783), SP (1:1000, anti-human guinea pig polyclonal, ABCAM Inc, Cambridge, England, ab10353), and NGF (1:20, anti-human goat polyclonal, R\&D Systems Inc, 614 McKineley PL NE Minneapolis, AF-256-NA). Alexa 488 donkey anti-mouse, Alexa Fluor 546 donkey antirabbit, Alexa Fluor 633 donkey anti-goat (ThermoFisher, Burlington, ON, Canada), and Alexa Fluor405 donkey anti-guinea pig (Sigma-Aldrich, MO, USA) at a concentration of 1:700 were used as corresponding fluorescent secondary antibodies for PGP 9.5, NR2B, NGF and SP, respectively. The antibody specificity was checked by removing the primary antibody. Images for the stained sections were taken by a Leica TCS SPE Confocal Microscope (Leica microsystems, Wetzlar, Germany). The analysis was performed blindly by a researcher who did not participate in biopsy collection or coding. To distinguish and count PGP 9.5 positive nerve fibers and to measure their area, the analysis and image processing program known as ImageJ (Image Processing and Analysis in Java; National Institutes of Health, USA) was used. The program was also used to identify nerve fibers (PGP9.5) coexpression with other molecules (SP, NR2B and NGF) ${ }^{36}$. Fibers were recognized as positive whenever PGP9.5 fluorescent signals were greater than +2 standard deviations (SDs) above the mean background of the image, and had a minimum width and length of $4 \mu \mathrm{m}^{11}$. PGP 9.5 positive nerve fibers were found in different tissues within the muscle; however, the current study looked only at the nerve fibers that were associated with myocytes and connective tissue. Hematoxylin staining was performed to facilitate description of cell types within the biopsies. Groups of tubular or round well-defined cells with multiple nuclei at the periphery were regarded as myocytes $^{37}$, while irregular tissue containing many cells and loose or dense fibers surrounding the myocytes was considered connective tissue ${ }^{38}$. Nerve fibers expressing SP were regarded as putative sensory afferent nerve fibers (Fig. 1b). The following formulas were used to calculate the density and expression frequency. Density equals 
a
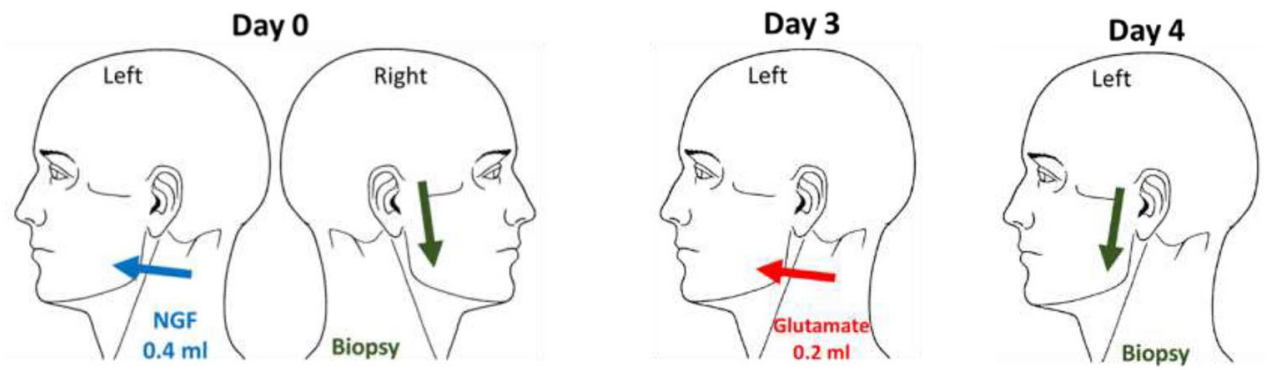

1

b p

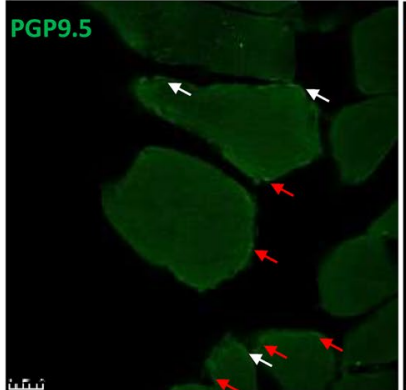

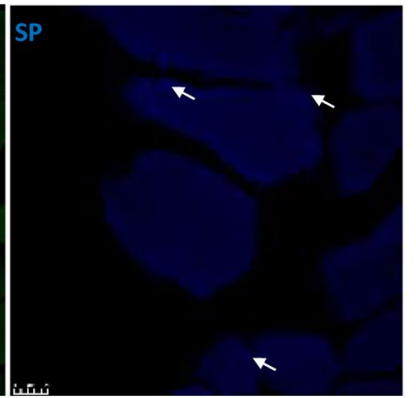

2
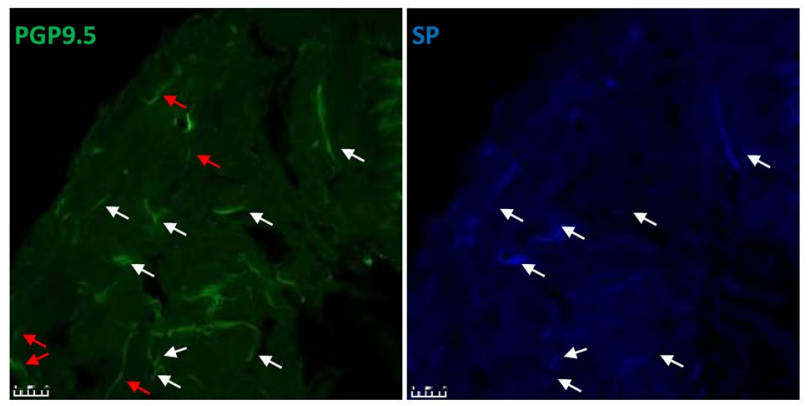

Figure 1. Figure displaying the methodology of the study. (a) Glutamate was injected three days after NGF into the left masseter muscle. One day after injection, a microbiopsy was taken from the injection site. (b) The left photomicrograph shows example image of PGP9.5 staining (green) while the right one shows SP expressing nerve fibers (blue) (1) associated with myocytes and (2) within connective tissue from one female participant on day 4. The white arrows indicate positive PGP 9.5 immunofluorescence signals co-expressed with positive SP signals, while the red arrows indicate positive PGP 9.5 signals without SP co-expression. Green: PGP 9.5, Blue: SP, Scale bar $=25 \mu \mathrm{m}$.

\begin{tabular}{|c|c|c|}
\hline & Men $(n=15)$ & Women $(n=15)$ \\
\hline \multicolumn{3}{|c|}{ Same tissue both days } \\
\hline Myocytes & 8 & 8 \\
\hline Connective tissue & 10 & 14 \\
\hline \multicolumn{3}{|l|}{ Tissue day 0} \\
\hline Myocytes & 8 & 8 \\
\hline Connective tissue & 12 & 14 \\
\hline \multicolumn{3}{|l|}{ Tissue day 4} \\
\hline Myocytes & 10 & 13 \\
\hline Connective tissue & 13 & 15 \\
\hline
\end{tabular}

Table 1. The number of participants. The table presents the number of participants whose biopsies contained myocytes and connective tissue on both days as well as the number of participants whose biopsies contained myocytes and connective tissue on day 0 and day 4 .

the number of positive fibers in a tissue divided by the total area of the same tissue on an image and averaged over the number of images per subject. Expression frequency equals the number of PGP9.5 positive fibers that were co-expressed with other substances divided by the total number of PGP9.5 positive fibers in the image and averaged over the number of images for the subject. Specific details on image analysis using Image J programme are previously explained in another study by the authors ${ }^{36}$.

Statistical analysis. Thirty participants were sufficient to test the hypothesis at a significant level of 0.05 and power of 0.80 , showing an estimated difference of $30 \%$ and a standard deviation of $50 \%$ in nerve fiber expression. Moreover, it has been proved that groups of 12 or more are enough to detect significant sex differences related to experimental pain model $s^{39,40}$. When data were compared between days, only data from participants whose biopsies carried the same tissue (connective tissue or myocytes) on both days were included in the analysis. However, when data were analyzed for correlations, all actual data from participants were included (Table 1). Significant differences and interaction between factors (sex and day) in the density and expression frequency of nerve fibers were detected by using a parametric 2-way RM ANOVA test; this was followed by posthoc comparisons using the Bonferroni-test. The Pearson or Spearman correlations (depending on whether data 


\begin{tabular}{|l|l|l|l|l|l|l|}
\hline & \multicolumn{3}{|l}{ All participants } & Men & \multicolumn{2}{l|}{ Women } \\
\cline { 2 - 7 } & Day 0 & Day 4 & Day 0 & Day 4 & Day 0 & Day 4 \\
\hline SP & $17(12)$ & $29(10)^{\# \#}$ & $14(10)$ & $23(10)$ & $20(14)$ & $35(6)^{\sharp *}$ \\
\hline NR2B & $89(17)$ & $88(9)$ & $93(3)$ & $91(5)$ & $85(24)$ & $85(11)$ \\
\hline NGF & $81(24)$ & $84(15)$ & $88(6)$ & $88(6)$ & $74(33)$ & $79(21)$ \\
\hline SP with NR2B & $16(12)$ & $27(10)^{\# \#}$ & $13(9)$ & $22(10)$ & $19(14)$ & $32(7)^{\#}$ \\
\hline SP with NGF & $16(12)$ & $25(9)^{\# \#}$ & $13(10)$ & $21(9)$ & $19(13)$ & $29(8)$ \\
\hline NR2B with NGF & $78(22)$ & $79(15)$ & $85(4)$ & $84(8)$ & $72(31)$ & $74(19)$ \\
\hline ALL & $15(11)$ & $24(8)^{\# \#}$ & $12(10)$ & $21(9)$ & $19(13)$ & $28(7)$ \\
\hline
\end{tabular}

Table 2. The frequency (\%) expression of nerve fibers associated with myocytes. The table presents the mean (SD) frequency of nerve fibers expressing markers alone and co-expressions (SP with NR2B, SP with NGF, NR2B with NGF, or All), from all participants, men, women on day 0 and 4 . All $=$ SP with NR2B and NGF. \# Significant differences between days in all participants (2-way RM ANOVA; P<0.05). "Significant differences between days (Bonferroni; $\mathrm{P}<0.05)$ within men or women. ${ }^{\star}$ Significant differences between men and women (Bonferroni; $\mathrm{P}<0.05$ ) within day 0 or day 4 .

was normally distributed) were used to examine the relationship between the peak pain intensity ( 5 min after glutamate injection on day 3) and the expression of SP, NGF and NR2B alone or their co-expressions (SP with NGF, SP with NR2B, NGF with NR2B, and all together) by nerve fibers on day 4, and also to test the relationship between the change (day 3 post glutamate injection/day 0 baseline) in mechanical pain characteristics and the expression of SP, NGF and NR2B on day 4 . The level of significance was set to $\mathrm{P}<0.05$. For statistical analysis, the SigmaPlot for Windows version 14.0 software (Systat Software Inc., San Jose, CA, USA) was used.

\section{Results}

The results of the combined effect of NGF and glutamate on the density of nerve fibers and expression of receptors and neuropeptides are presented. The difference between connective tissue and myocytes on density as well as on nerve fibers expression has been presented elsewhere ${ }^{36}$.

The combined effect of NGF and glutamate on the density of nerve fibers. The average density of PGP 9.5 positive nerve fibers associated with myocytes did not differ between days $(\mathrm{F}=0.842, \mathrm{P}=0.374)$ or $\operatorname{sex}(\mathrm{F}=0.063, \mathrm{P}=0.805)$. However, the density of PGP 9.5 positive nerve fibers expressing $\mathrm{SP}$ was significantly greater on day 4 compared to day $0(\mathrm{~F}=6.970, \mathrm{P}=0.019)$. No sex-related differences were detected $(\mathrm{F}=1.459$, $\mathrm{P}=0.247)$. No significant interaction was found between factors $(\mathrm{F}=0.078, \mathrm{P}=0.783)$.

There were no significant differences in the average density of PGP 9.5 positive nerve fibers between days $(\mathrm{F}=0.494, \mathrm{P}=0.489)$ or between sexes $(\mathrm{F}=0.100, \mathrm{P}=0.754)$ within connective tissue. No significant differences in the average density of PGP 9.5 positive nerve fibers expressing SP between days $(F=0.871, P=0.361)$ or between sexes $(\mathrm{F}=1.504, \mathrm{P}=0.233)$ were identified.

The combined effect of injections on the expression of receptors and neuropeptides. Myocytes. Analyzed data from all participants showed an increase in the frequency of nerve fiber expression of SP alone $(\mathrm{F}=13.713, \mathrm{P}=0.002)$, with $\mathrm{NR} 2 \mathrm{~B}(\mathrm{~F}=10.599, \mathrm{P}=0.006)$ and with $\mathrm{NGF}(\mathrm{F}=5.151, \mathrm{P}=0.040)$ as well as all three markers together $(\mathrm{F}=4.774, \mathrm{P}=0.046)$ on day 4 compared to day 0 . No significant differences were detected between days in nerve fiber expression of NR2B or NGF alone or both of them in combination (Table 2). There were no significant differences in the expression frequency of SP, NR2B, or NGF between sexes. However, within day 4 , the frequency of nerve fibers expressing SP alone $(\mathrm{P}=0.032)$ was significantly higher in women compared with men (Table 2).

Connective tissue. When data from all participants were used for analysis, no significant changes in the frequency of expression of SP, NR2B, or NGF by nerve fibers were detected between days (Table 3). However, the nerve fiber expression of SP $(\mathrm{F}=6.296, \mathrm{P}=0.020)$, NR2B $(\mathrm{F}=4.956, \mathrm{P}=0.037)$, SP with NR2B $(\mathrm{F}=8.366$, $\mathrm{P}=0.008)$, SP with NGF $(\mathrm{F}=4.375, \mathrm{P}=0.048)$ and all three markers together $(\mathrm{F}=4.716, \mathrm{P}=0.041)$ was significantly higher in women when compared with men.

The correlation between the expression of putative sensory afferent nerve fibers and mechanical sensitivity induced by NGF and glutamate. Myocytes. There was a significant positive correlation between the nerve fiber expression of SP alone and the percentage change in temporal summation pain (day 3 post glutamate injection/day 0 baseline) $(r=0.415, n=23, P=0.048)$. The nerve fiber expression of SP in combination with NR2B was also correlated with the percentage change in temporal summation pain $(\mathrm{r}=0.437$, $\mathrm{n}=23, \mathrm{P}=0.036$ ). No significant correlation was found for other mechanical sensitivity parameters (PPT and chewing-evoked pain) $(\mathrm{P}>0.05)$. 


\begin{tabular}{|l|l|l|l|l|l|l|}
\hline & \multicolumn{4}{|l}{ All participants } & Men & \multicolumn{2}{l|}{ Women } \\
\cline { 2 - 8 } & Day 0 & Day 4 & Day 0 & Day 4 & Day 0 & Day 4 \\
\hline SP & $61(14)$ & $58(17)$ & $56(14)$ & $48(19)$ & $64(13)$ & $64(13)^{\star}$ \\
\hline NR2B & $57(21)$ & $62(19)$ & $48(15)$ & $53(13)$ & $64(23)^{\star}$ & $69(20)$ \\
\hline NGF & $26(19)$ & $27(13)$ & $25(17)$ & $24(13)$ & $27(20)$ & $29(13)$ \\
\hline SP with NR2B & $38(19)$ & $40(18)$ & $27(11)$ & $29(18)$ & $45(20)^{\star}$ & $47(15)^{\star}$ \\
\hline SP with NGF & $14(10)$ & $16(10)$ & $11(7)$ & $11(6)$ & $16(11)$ & $19(11)$ \\
\hline NR2B with NGF & $22(18)$ & $25(13)$ & $20(14)$ & $22(12)$ & $24(21)$ & $27(13)$ \\
\hline ALL & $13(9)$ & $15(9)$ & $9(7)$ & $10(6)$ & $15(10)$ & $18(12)$ \\
\hline
\end{tabular}

Table 3. The frequency (\%) expression of nerve fibers within connective tissue. The table presents the mean (SD) frequency of nerve fibers expressing markers alone and co-expressions (SP with NR2B, SP with NGF, NR2B with NGF, or All), from all participants, men, women on day 0 and 4 . All $=$ SP with NR2B and NGF. ${ }^{*}$ Significant differences between men and women (Bonferroni; $\mathrm{P}<0.05$ ) within day 0 or day 4 .
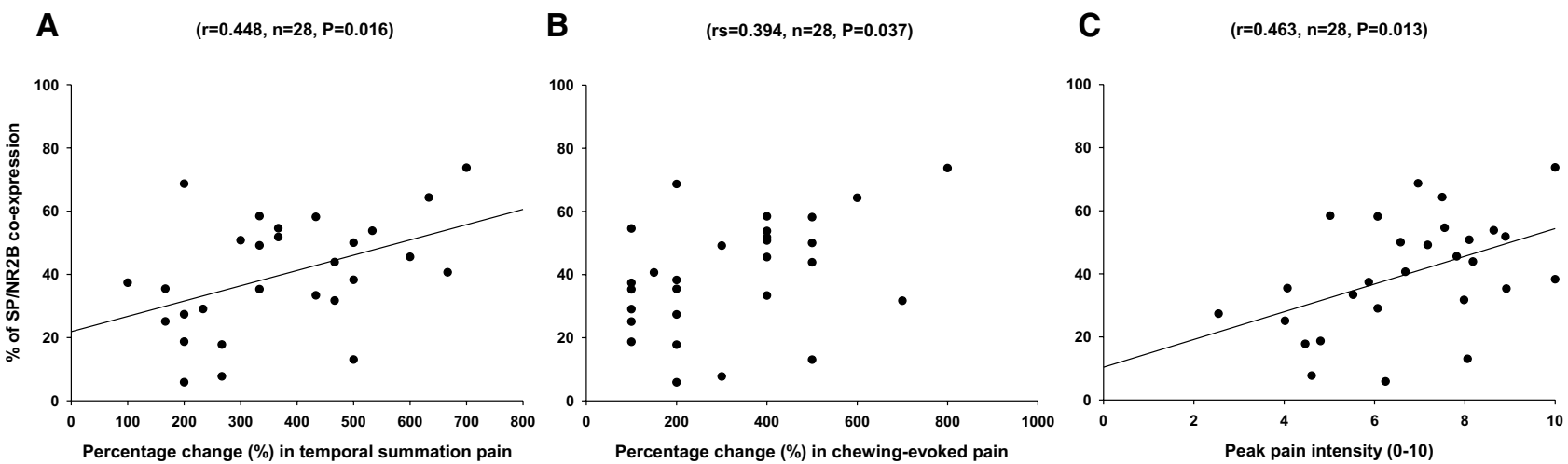

Figure 2. The scatter plot illustrates correlations between different pain characteristics and NR2B receptor expression by putative sensory afferent nerve fibers. The correlation between the co-expression of SP with NR2B by nerve fibers and the percentage change (day 3 post glutamate injection/day 0 baseline) in (A) temporal summation pain and (B) chewing-evoked pain. $(\mathbf{C})$ is presenting the correlation of SP with NR2B co-expression with the peak pain intensity induced by injection of glutamate three days after NGF administration. $r$ Pearson, $r s$ Spearman correlation coefficient.

Connective tissue. Data from all participants showed significant positive correlations between the expression of SP with NR2B by nerve fibers on day 4 and the percentage change (day 3 post glutamate injection/day 0 baseline) in temporal summation pain $(\mathrm{r}=0.448, \mathrm{n}=28, \mathrm{P}=0.016)$ (Fig. $2 \mathrm{~A})$ as well as chewing-evoked pain $\left(\mathrm{r}_{\mathrm{s}}=0.394\right.$, $\mathrm{n}=28, \mathrm{P}=0.037$ ) (Fig. 2B). No significant correlations were found for PPT.

The correlation between the expression of SP with NR2B and pain induced by glutamate. $\quad M y$ ocytes. No significant correlations were found.

Connective tissue. A significant positive correlation was detected between the nerve fiber expression of SP with $\mathrm{NR} 2 \mathrm{~B}$ and the peak glutamate-evoked pain intensity in all participants $(\mathrm{r}=0.463, \mathrm{n}=28, \mathrm{P}=0.013)$ (Fig. 2C). However, no significant correlation was found when data analyzed from men $(\mathrm{r}=0.315, \mathrm{n}=13, \mathrm{P}=0.294)$ and women $(\mathrm{r}=0.495, \mathrm{n}=15, \mathrm{P}=0.060)$ were undertaken separately.

\section{Discussion}

The main findings of the current study were that: (1) the combination of NGF and glutamate injection into the masseter muscle increases the density and expression of putative sensory afferent nerve fibers that express SP; (2) the density of putative sensory afferent nerve fibers does not differ between sexes, but the expression of putative sensory afferent nerve fibers is greater in women than men. (3) The increase in temporal summation pain and chewing-evoked pain induced by NGF and glutamate is positively correlated with the expression of NR2B by putative sensory nerve fibers; and (4) peak glutamate-induced pain intensity is positively correlated with expression of NR2B by putative sensory nerve fibers in NGF-sensitized masseter muscle.

$\mathrm{SP}$ is an important peptide that is involved in many physiological and pathophysiological processes, including nociception ${ }^{41}$. At the level of the trigeminal ganglion, SP was mostly found in unmyelinated small diameter nerve fibers ${ }^{42}$ which comprise $10-30 \%$ of all trigeminal nerve fibers ${ }^{43}$. SP expressing sensory afferent nerve fibers project to the brainstem trigeminal sensory nucleus, and upon release increase the excitability of trigeminal sensory 
neurons ${ }^{44,45}$ Injury induced by extraction of maxillary molars increases the number of SP immunoreactive neurons in the trigeminal ganglion ${ }^{46}$. Moreover, NGF increases the expression of SP by masseter ganglion neurons in female rats ${ }^{11}$. In rats, it has been shown that NGF can increase the level of SP at the dorsal root ganglion ${ }^{49}$. Thus, NGF contributes to central sensitization partly by increasing the release of neuropeptides including $\mathrm{SP}^{47}$. The current study is the first, to our knowledge, to show an increase in the frequency of nerve fiber expression of SP as a consequence of NGF and glutamate injections into human muscles. This finding may suggest the involvement of this nociceptive peptide in peripheral sensitization. However, a recent published article has demonstrated lack of changes of plasma SP levels in patients with TMD compared to healthy controls ${ }^{32}$. Moreover, injection of SP into the temporalis muscle was not painful, and into the tibialis muscle failed to produce hyperalgesia to $\mathrm{PPT}^{48,49}$. Large-scale trials targeting SP-antagonism in humans failed to successfully reduce pain ${ }^{50}$. These contradictory results could be attributed to differences between rats and humans in terms of the contribution of SP to pain mechanisms, as in humans, SP can contribute to other biological processes such as vasodilatation and inflammation ${ }^{51,52}$. It is still possible that SP acts to enhance glutamate-induced mechanical sensitization of masticatory muscle in humans ${ }^{49,53}$.

There is some evidence which suggests that NGF modulates both the expression and function of NMDA receptors to alter the response properties of masseter muscle sensory afferent nerve fibers. NGF injection into the masseter muscle of healthy participants induced mechanical sensitization that lasted up to 3 weeks ${ }^{13,16}$. In rats, there is evidence that NGF-induced masseter muscle sensitization is caused by increased expression of NMDA receptors by sensory afferent fibers ${ }^{11}$. Injection of glutamate can also evoke masseter muscle sensitization through the activation of peripheral NMDA receptors ${ }^{12,54}$. Injection of glutamate into masseter muscles pretreated with NGF failed to cause an increase or decrease in $\mathrm{PPT}^{15}$, which could be an indication that glutamate and NGF can share a common pathway, thus preventing additional sensitization when these substances are used together ${ }^{13}$. It is also possible that a ceiling effect occurred by NGF on PPT and that additional sensitization by glutamate, thus was not possible. The current study showed that experimental myalgia induced by NGF and glutamate can increase the density of masseter sensory afferent nerve fibers as well as the expression of NR2B and NGF. This finding supports the suggested interaction of NGF and NMDA receptors ${ }^{11}$ and may indicate their involvement in the peripheral mechanism that underlies muscle sensitization.

Earlier studies showed that an injection of NGF into the masseter muscle reduced PPT more in women than in men $^{16,55}$. The reduction of PPT in women was associated with an increased expression of NMDA-receptors by sensory afferent nerve fibers ${ }^{56}$. It has also been reported that injection of glutamate into the masseter muscle produces more intense pain in women than in men $^{40,57}$. In female rats, increased estrogen levels are associated with higher expression of NR2B subunit-containing NMDA-receptors by masseter ganglion neurons and an increased sensory afferent nerve fiber discharge evoked by injection of NMDA into the masseter muscle ${ }^{54}$. NGF-induced mechanical sensitization was suggested to be due to the greater co-expression of SP with NR2B in masseter ganglion neurons of female as compared with male rats ${ }^{11}$. Consistent with animal reports, the current study showed sex differences related to the putative sensory afferent nerve fiber expression of NR2B subunitcontaining NMDA-receptors as well as NGF. Hence, one can speculate that the sex-related differences in NGF induced muscle sensitization and glutamate evoked muscle pain are similar in rats and humans, and thereby also attributable to the sensory afferent nerve fiber expression of NR2B and NGF. However, the present study found no association between the expression of NMDA-receptors by nerve fibers and the change in PPT in women. The lack of association in the current study compared to our previous study ${ }^{56}$, is not unexpected as no sex-related differences in masseter muscle sensitization were detected post injection of glutamate into the masseter muscle ${ }^{8,40}$.

In a previous study we have presented a positive correlation between peripheral nerve fibers expression of NMDA receptors and pain characteristics after glutamate injection into the masseter muscle ${ }^{56}$. The present experimental pain model also demonstrates a significant association between the nerve fiber expression of peripheral NMDA-receptors and pain characteristics (pain intensity, chewing-evoked pain and temporal summation pain) reminiscent of pain complaints in individuals with TMD myalgia. Masticatory muscle pain and fatigue induced by chewing are common signs and symptoms of TMD myalgia ${ }^{58}$. Peak pain intensity reported by healthy participants after injection of glutamate into the masseter muscle is similar to the peak pain reported by patients with $\mathrm{TMD}^{7}$. Temporal summation of fixed mechanical stimulation can reflect central sensitization in an experimental pain model, and has been shown to be dependent on the activation of NMDA receptors ${ }^{59}$. Temporal summation pain reported by patients with TMD was higher than in healthy controls ${ }^{60,61}$. These findings together strengthen the theory that NMDA-receptors expressed peripherally by sensory afferent fibers and/ or in the central nervous system plays a role with regard to the pathogenesis of TMD myalgia ${ }^{12,33,62}$, and that increasing NMDA receptor expression may cause the muscle to be more sensitive to pain.

A possible limitation for this study is that the number of sensory afferent fibers within muscle tissues was likely underestimated, as the expression of other neuropeptides associated with sensory afferent fibers was not assessed. For example, calcitonin gene-related peptide (CGRP) is thought to play an important role in nociception $^{63}$ and is highly expressed by masseter muscle sensory afferent nerve fibers in rats ${ }^{11}$. Indeed, coexpression of CGRP and NMDA receptors was also increased in female rats after masseter muscle injection of NGF. If NGF exerts a similar effect on these sensory fibers in humans, then our study also likely underestimated the increase in NMDA expression that could be induced by NGF. Unfortunately, at the time this study was conducted, there was no available CGRP antibody compatible with the NMDA and PGP 9.5 antibodies for humans. Future studies will be required to address this question. 


\section{Conclusions}

Injections of NGF or glutamate are useful experimental models for the investigation of TMD myalgia ${ }^{16}$. The current study has demonstrated that cellular and molecular changes occur after combined injection of glutamate and NGF into the human masseter muscle. The combined injections increased the density of SP expressing nerve fibers as well as the expression of NMDA receptors and NGF by putative sensory afferent nerve fibers. This increase in expression was significantly greater in women than in men. Further, the expression of NMDA-receptors by putative sensory afferent nerve fibers was significantly associated with increased pain during functional activity and increased muscle pain sensitivity in both men and women. Hence, and in accordance with previous animal findings $s^{11,12}$ the current human study can conclude that NMDA-receptors and NGF expressed by peripheral putative sensory afferent nerve fibers seem to play an important role in the mechanisms of muscle sensitization and sex-related differences in pain reports associated with this model of masseter muscle myalgia. However, whether these results are relevant to the mechanisms of clinical muscle pain, for example, in myofascial TMD, is an open question that warrants further investigation.

\section{Data availability}

The datasets generated during and/or analyzed during the current study are available from the corresponding author on reasonable request.

Received: 7 April 2021; Accepted: 16 July 2021

Published online: 02 August 2021

\section{References}

1. LeResche, L. Epidemiology of temporomandibular disorders: Implications for the investigation of etiologic factors. Crit. Rev. Oral Biol. Med. Off. Publ. Am. Assoc. Oral Biol. 8, 291-305 (1997).

2. Schiffman, E. et al. Diagnostic criteria for temporomandibular disorders (DC/TMD) for clinical and research applications: Recommendations of the International RDC/TMD Consortium Network* and Orofacial Pain Special Interest Groupdagger. J. Oral Facial Pain Headache 28, 6-27. https://doi.org/10.11607/jop.1151 (2014).

3. Isong, U., Gansky, S. A. \& Plesh, O. Temporomandibular joint and muscle disorder-type pain in US adults: The National Health Interview Survey. J. Orofacial Pain 22, 317-322 (2008).

4. Nilsson, I. M., List, T. \& Drangsholt, M. Incidence and temporal patterns of temporomandibular disorder pain among Swedish adolescents. J. Orofac. Pain 21, 127-132 (2007).

5. Arendt-Nielsen, L. \& Graven-Nielsen, T. Muscle pain: Sensory implications and interaction with motor control. Clin. J. Pain 24, 291-298. https://doi.org/10.1097/AJP.0b013e31815b608f (2008).

6. Svensson, P. \& Graven-Nielsen, T. Craniofacial muscle pain: Review of mechanisms and clinical manifestations. J. Orofac. Pain 15, 117-145 (2001).

7. Castrillon, E. E. et al. Glutamate-evoked jaw muscle pain as a model of persistent myofascial TMD pain?. Arch. Oral Biol. 53, 666-676. https://doi.org/10.1016/j.archoralbio.2008.01.008 (2008).

8. Cairns, B. E., Gambarota, G., Svensson, P., Arendt-Nielsen, L. \& Berde, C. B. Glutamate-induced sensitization of rat masseter muscle fibers. Neuroscience 109, 389-399 (2002).

9. Christidis, N., Ioannidou, K., Milosevic, M., Segerdahl, M. \& Ernberg, M. Changes of hypertonic saline-induced masseter muscle pain characteristics, by an infusion of the serotonin receptor type 3 antagonist granisetron. J. Pain Off. J. Am. Pain Soc. 9, 892-901. https://doi.org/10.1016/j.jpain.2008.05.002 (2008).

10. Ernberg, M., Lundeberg, T. \& Kopp, S. Effect of propranolol and granisetron on experimentally induced pain and allodynia/ hyperalgesia by intramuscular injection of serotonin into the human masseter muscle. Pain 84, 339-346 (2000).

11. Wong, H. et al. NGF-induced mechanical sensitization of the masseter muscle is mediated through peripheral NMDA receptors. Neuroscience 269, 232-244. https://doi.org/10.1016/j.neuroscience.2014.03.054 (2014).

12. Cairns, B. E. et al. Activation of peripheral NMDA receptors contributes to human pain and rat afferent discharges evoked by injection of glutamate into the masseter muscle. J. Neurophysiol. 90, 2098-2105. https://doi.org/10.1152/jn.00353.2003 (2003).

13. Svensson, P., Wang, K., Arendt-Nielsen, L. \& Cairns, B. E. Effects of NGF-induced muscle sensitization on proprioception and nociception. Exp. Brain Res. 189, 1-10. https://doi.org/10.1007/s00221-008-1399-4 (2008).

14. Louca, S., Ernberg, M. \& Christidis, N. Influence of intramuscular granisetron on experimentally induced muscle pain by acidic saline. J. Oral Rehabil. 40, 403-412. https://doi.org/10.1111/joor.12046 (2013).

15. Shimada, A., Alhilou, A. M., Svensson, P., Ernberg, M. \& Christidis, N. Functional change in experimental allodynia after glutamate-induced pain in the human masseter muscle. Front. Oral Health https://doi.org/10.3389/froh.2020.609082 (2020).

16. Svensson, P., Cairns, B. E., Wang, K. \& Arendt-Nielsen, L. Injection of nerve growth factor into human masseter muscle evokes long-lasting mechanical allodynia and hyperalgesia. Pain 104, 241-247 (2003).

17. Woolf, C. J., Safieh-Garabedian, B., Ma, Q. P., Crilly, P. \& Winter, J. Nerve growth factor contributes to the generation of inflammatory sensory hypersensitivity. Neuroscience 62, 327-331. https://doi.org/10.1016/0306-4522(94)90366-2 (1994).

18. Björkman, R., Hallman, K. M., Hedner, J., Hedner, T. \& Henning, M. Acetaminophen blocks spinal hyperalgesia induced by NMDA and substance P. Pain 57, 259-264. https://doi.org/10.1016/0304-3959(94)90001-9 (1994).

19. Steiner, P., Pfeilschifter, J., Boeckh, C., Radeke, H. \& Otten, U. Interleukin-1 beta and tumor necrosis factor-alpha synergistically stimulate nerve growth factor synthesis in rat mesangial cells. Am. J. Physiol. 261, F792-798. https://doi.org/10.1152/ajprenal.1991. 261.5.F792 (1991).

20. März, P., Heese, K., Dimitriades-Schmutz, B., Rose-John, S. \& Otten, U. Role of interleukin-6 and soluble IL-6 receptor in regionspecific induction of astrocytic differentiation and neurotrophin expression. Glia 26, 191-200. https://doi.org/10.1002/(sici) 10981136(199905)26:3\%3c191::aid-glia1\%3e3.0.co;2-\# (1999).

21. Freund, V. et al. Upregulation of nerve growth factor expression by human airway smooth muscle cells in inflammatory conditions. Eur. Respir. J. 20, 458-463. https://doi.org/10.1183/09031936.02.00269202 (2002).

22. Manni, L. et al. Nerve growth factor release by human synovial fibroblasts prior to and following exposure to tumor necrosis factoralpha, interleukin-1 beta and cholecystokinin-8: The possible role of NGF in the inflammatory response. Clin. Exp. Rheumatol. 21, 617-624 (2003).

23. von Boyen, G. B. et al. Nerve growth factor secretion in cultured enteric glia cells is modulated by proinflammatory cytokines. J. Neuroendocrinol. 18, 820-825. https://doi.org/10.1111/j.1365-2826.2006.01478.x (2006).

24. Woolf, C. J. Phenotypic modification of primary sensory neurons: The role of nerve growth factor in the production of persistent pain. Philos. Trans. R. Soc. Lond. Series B Biol. Sci. 351, 441-448. https://doi.org/10.1098/rstb.1996.0040 (1996). 
25. Goedert, M., Stoeckel, K. \& Otten, U. Biological importance of the retrograde axonal transport of nerve growth factor in sensory neurons. Proc. Natl. Acad. Sci. USA 78, 5895-5898. https://doi.org/10.1073/pnas.78.9.5895 (1981).

26. Reinert, A., Kaske, A. \& Mense, S. Inflammation-induced increase in the density of neuropeptide-immunoreactive nerve endings in rat skeletal muscle. Exp. Brain Res. 121, 174-180. https://doi.org/10.1007/s002210050449 (1998).

27. Rukwied, R. et al. NGF induces non-inflammatory localized and lasting mechanical and thermal hypersensitivity in human skin. Pain 148, 407-413. https://doi.org/10.1016/j.pain.2009.11.022 (2010).

28. Singer, E. \& Dionne, R. A controlled evaluation of ibuprofen and diazepam for chronic orofacial muscle pain. J. Orofac. Pain 11, 139-146 (1997).

29. Zarb, G. A., Carlsson, G. E., Sessle, B. J. \& Mohl, N. D. Temporomandibular Joint and Mastication Muscular Disorders (WileyBlackwell, 1995).

30. Cairns, B. E. Pathophysiology of TMD pain-basic mechanisms and their implications for pharmacotherapy. J. Oral Rehabil. 37, 391-410. https://doi.org/10.1111/j.1365-2842.2010.02074.x (2010).

31. Castrillon, E. E. et al. Interstitial glutamate concentration is elevated in the masseter muscle of myofascial temporomandibular disorder patients. J. Orofac. Pain 24, 350-360 (2010).

32. Jasim, H., Ghafouri, B., Gerdle, B., Hedenberg-Magnusson, B. \& Ernberg, M. Altered levels of salivary and plasma pain related markers in temporomandibular disorders. J. Headache Pain 21, 105. https://doi.org/10.1186/s10194-020-01160-z (2020).

33. Cairns, B. E. et al. Ketamine attenuates glutamate-induced mechanical sensitization of the masseter muscle in human males. Exp. Brain Res. 169, 467-472. https://doi.org/10.1007/s00221-005-0158-z (2006).

34. Svensson, P., Arendt-Nielsen, L., Nielsen, H. \& Larsen, J. K. Effect of chronic and experimental jaw muscle pain on pain-pressure thresholds and stimulus-response curves. J. Orofac. Pain 9, 347-356 (1995).

35. Christidis, N. et al. Expression of 5-HT3 receptors and TTX resistant sodium channels (NaV1.8) on muscle nerve fibers in painfree humans and patients with chronic myofascial temporomandibular disorders. J. Headache Pain 15, 63. https://doi.org/10.1186/ 1129-2377-15-63 (2014).

36. Alhilou, A. et al. Density of nerve fibers and expression of substance P, NR2B-receptors, and nerve growth factor in healthy human masseter muscle: An immunohistochemical study. J. Oral Rehabil. https://doi.org/10.1111/joor.13109 (2020).

37. Frontera, W. R. \& Ochala, J. Skeletal muscle: A brief review of structure and function. Calcif. Tissue Int. 96, 183-195. https://doi. org/10.1007/s00223-014-9915-y (2015).

38. Corcoran, N. M. \& Goldman, E. M. StatPearls (StatPearls Publishing LLC, 2020).

39. Cairns, B. E., Hu, J. W., Arendt-Nielsen, L., Sessle, B. J. \& Svensson, P. Sex-related differences in human pain and rat afferent discharge evoked by injection of glutamate into the masseter muscle. J. Neurophysiol. 86, 782-791 (2001).

40. Svensson, P. et al. Glutamate-evoked pain and mechanical allodynia in the human masseter muscle. Pain 101, 221-227 (2003).

41. Mashaghi, A. et al. Neuropeptide substance P and the immune response. Cell. Mol. Life Sci. CMLS 73, 4249-4264. https://doi.org/ 10.1007/s00018-016-2293-z (2016).

42. Bae, J. Y., Kim, J. H., Cho, Y. S., Mah, W. \& Bae, Y. C. Quantitative analysis of afferents expressing substance P, calcitonin generelated peptide, isolectin B4, neurofilament 200, and Peripherin in the sensory root of the rat trigeminal ganglion. J. Comp. Neurol. 523, 126-138. https://doi.org/10.1002/cne.23672 (2015).

43. Lehtosalo, J. I., Uusitalo, H., Stjernschantz, J. \& Palkama, A. Substance P-like immunoreactivity in the trigeminal ganglion. A fluorescence, light and electron microscope study. Histochemistry 80, 421-427. https://doi.org/10.1007/bf00495429 (1984).

44. Cuello, A. C., Del Fiacco, M. \& Paxinos, G. The central and peripheral ends of the substance P-containing sensory neurones in the rat trigeminal system. Brain Res. 152, 499-500. https://doi.org/10.1016/0006-8993(78)91105-8 (1978).

45. Henry, J. L., Sessle, B. J., Lucier, G. E. \& Hu, J. W. Effects of substance P on nociceptive and non-nociceptive trigeminal brain stem neurons. Pain 8, 33-45. https://doi.org/10.1016/0304-3959(80)90088-3 (1980).

46. Goto, T., Iwai, H., Kuramoto, E. \& Yamanaka, A. Neuropeptides and ATP signaling in the trigeminal ganglion. Japan. Dental Sci. Rev. 53, 117-124. https://doi.org/10.1016/j.jdsr.2017.01.003 (2017).

47. Mendell, L. M., Albers, K. M. \& Davis, B. M. Neurotrophins, nociceptors, and pain. Microsc. Res. Tech. 45, 252-261. https://doi. org/10.1002/(sici)1097-0029(19990515/01)45:4/5\%3c252::aid-jemt9\%3e3.0.co;2-n (1999).

48. Babenko, V. V. et al. Experimental human muscle pain induced by intramuscular injections of bradykinin, serotonin, and substance P. Eur. J. Pain (London, England) 3, 93-102. https://doi.org/10.1053/eujp.1998.0103 (1999).

49. Jensen, K., Tuxen, C., Pedersen-Bjergaard, U. \& Jansen, I. Pain, tenderness, wheal and flare induced by substance-P, bradykinin and 5-hydroxytryptamine in humans. Cephalalgia Int. J. Headache 11, 175-182. https://doi.org/10.1046/j.1468-2982.1991.11041 75.x (1991).

50. Steinhoff, M. S., von Mentzer, B., Geppetti, P., Pothoulakis, C. \& Bunnett, N. W. Tachykinins and their receptors: Contributions to physiological control and the mechanisms of disease. Physiol. Rev. 94, 265-301. https://doi.org/10.1152/physrev.00031.2013 (2014).

51. Bull, H. A., Hothersall, J., Chowdhury, N., Cohen, J. \& Dowd, P. M. Neuropeptides induce release of nitric oxide from human dermal microvascular endothelial cells. J. Invest. Dermatol. 106, 655-660. https://doi.org/10.1111/1523-1747.ep12345471 (1996).

52. O'Connor, T. M. et al. The role of substance P in inflammatory disease. J. Cell. Physiol. 201, 167-180. https://doi.org/10.1002/jcp. 20061 (2004).

53. Jensen, K. et al. Pain and tenderness in human temporal muscle induced by bradykinin and 5-hydroxytryptamine. Peptides 11, 1127-1132. https://doi.org/10.1016/0196-9781(90)90141-q (1990).

54. Dong, X. D. et al. Sex-related differences in NMDA-evoked rat masseter muscle afferent discharge result from estrogen-mediated modulation of peripheral NMDA receptor activity. Neuroscience 146, 822-832. https://doi.org/10.1016/j.neuroscience.2007.01. 051 (2007).

55. Svensson, P., Castrillon, E. \& Cairns, B. E. Nerve growth factor-evoked masseter muscle sensitization and perturbation of jaw motor function in healthy women. J. Orofac. Pain 22, 340-348 (2008).

56. Alhilou, A. M. et al. Sex-related differences in response to masseteric injections of glutamate and nerve growth factor in healthy human participants. Sci. Rep. 11, 13873. https://doi.org/10.1038/s41598-021-93171-2 (2021).

57. Castrillon, E. E., Cairns, B. E., Wang, K., Arendt-Nielsen, L. \& Svensson, P. Comparison of glutamate-evoked pain between the temporalis and masseter muscles in men and women. Pain 153, 823-829. https://doi.org/10.1016/j.pain.2012.01.003 (2012).

58. Fernández-de-las-Penas, C. \& Svensson, P. Myofascial temporomandibular disorder. Curr. Rheumatol. Rev. 12, 40-54. https://doi. org/10.2174/1573397112666151231110947 (2016).

59. Eide, P. K. Wind-up and the NMDA receptor complex from a clinical perspective. Eur. J. Pain (London, England) 4, 5-15. https:// doi.org/10.1053/eujp.1999.0154 (2000).

60. Sarlani, E., Garrett, P. H., Grace, E. G. \& Greenspan, J. D. Temporal summation of pain characterizes women but not men with temporomandibular disorders. J. Orofac. Pain 21, 309-317 (2007).

61. Sarlani, E., Grace, E. G., Reynolds, M. A. \& Greenspan, J. D. Evidence for up-regulated central nociceptive processing in patients with masticatory myofascial pain. J. Orofac. Pain 18, 41-55 (2004).

62. Cairns, B. E. et al. Systemic administration of monosodium glutamate elevates intramuscular glutamate levels and sensitizes rat masseter muscle afferent fibers. Pain 132, 33-41. https://doi.org/10.1016/j.pain.2007.01.023 (2007).

63. Schou, W. S., Ashina, S., Amin, F. M., Goadsby, P. J. \& Ashina, M. Calcitonin gene-related peptide and pain: A systematic review. J. Headache Pain 18, 34. https://doi.org/10.1186/s10194-017-0741-2 (2017). 


\section{Author contributions}

A.A.: Performed the research, analyzed the data and drafted the manuscript. A.S.: Collected the research data, participated in the research design and manuscript editing. C.S.: Participated in data analysis and manuscript editing. P.S.: Participated in data collection and manuscript editing. M.E.: Participated in the research design and manuscript editing. B.E.C.: Participated in the research design, data analysis and manuscript editing. N.C.: Designed the research, participated in data analysis and manuscript editing.

\section{Funding}

Open access funding provided by Karolinska Institute.

\section{Competing interests}

The authors declare no competing interests.

\section{Additional information}

Correspondence and requests for materials should be addressed to A.M.A.

Reprints and permissions information is available at www.nature.com/reprints.

Publisher's note Springer Nature remains neutral with regard to jurisdictional claims in published maps and institutional affiliations.

(c) (i) Open Access This article is licensed under a Creative Commons Attribution 4.0 International License, which permits use, sharing, adaptation, distribution and reproduction in any medium or format, as long as you give appropriate credit to the original author(s) and the source, provide a link to the Creative Commons licence, and indicate if changes were made. The images or other third party material in this article are included in the article's Creative Commons licence, unless indicated otherwise in a credit line to the material. If material is not included in the article's Creative Commons licence and your intended use is not permitted by statutory regulation or exceeds the permitted use, you will need to obtain permission directly from the copyright holder. To view a copy of this licence, visit http://creativecommons.org/licenses/by/4.0/.

(C) The Author(s) 2021 